Florentino Serranheira ${ }^{1,2}$

António de Sousa Uva ${ }^{1,3}$

Jorge Espírito-Santo ${ }^{4}$

\section{Estratégia de avaliação do risco de lesões músculo- esqueléticas de membros superiores ligadas ao tra- balho aplicada na indústria de abate e desmancha de carne em Portugal ${ }^{*, * *}$}

\author{
Work-related upper limbs musculoskeletal disorder risk evaluation \\ strategy in a Portuguese meatpacking plant
}

\section{Resumo}

Objectivo: As lesões músculo-esqueléticas ligadas ao trabalho (LMELT) são doenças profissionais frequentes. Neste estudo ensaiou-se uma estratégia de diagnóstico do risco e de vigilância da saúde dos trabalhadores numa empresa de abate e desmancha de carne. Métodos: Utilizou-se uma estratégia de obtenção de informação em todos os postos de trabalho e trabalhadores da empresa. Utilizaram-se: (i) adaptação do Questionário Nórdico Músculo-Esquelético, incluindo caracterização da exposição biomecânica; (ii) protocolo de avaliação clínica de LMELT; (iii) filtro RSI e método Strain Index; (iv) instrumentação, como electrogoniometria e sensores de força em postos de risco elevado. Resultados: Identificou-se a presença de sintomas e sinais de LMELT principalmente nos punhos/mãos $(n=27)$ e região lombo-sagrada $(\mathrm{n}=32)$, uma importante prevalência de casos relacionados com a actividade de trabalho (30\%) e níveis de risco elevados com base nas classificações do Strain Index ( $\mathrm{n}=26$ MSDto e $\mathrm{n}=7$ MSEsq). A utilização da instrumentação permitiu obter detalhes da repetitividade, das posturas e dos momentos de aplicação de força, úteis para a intervenção. Conclusões: A prevenção só é possível através da aplicação de programas/estratégias integradas de diagnóstico e gestão do risco de LMELT que sejam eficazes no sentido da intervenção sobre a actividade e as condições de trabalho.

Palavras-chave: Saúde Ocupacional, Ergonomia, Lesões Músculo-Esqueléticas Ligadas ao Trabalho (LMELT), LER/DORT, abate e desmancha de carnes, avaliação e gestão de riscos profissionais. \begin{abstract}
Background: Work-related Musculoskeletal Disorders (WRMSD) are common occupational diseases. The present study aims at examining an integrated perspective of risk assessment and health surveillance at a meatpacking plant. Methods: The strategy adopted was of obtaining information about WRMSDs awareness at all workstations and from all their workers. This was based on: (i) questionnaire application - an adaptation of the Nordic musculoskeletal questionnaire, including a biomechanical item, (ii) WRMSDs clinical protocol (iii) RSI risk filter and Strain Index application, (iv) instrumentation with electrogoniometry and force sensors at previously classified as high risk workstations. Results: WRMSDs signs and symptoms mainly in wrist/hands $(n=27)$ and in lumbar region $(n=32)$ were identified. Results revealed an important prevalence of WRULMSDs associated to meatpacking industry activities (30\%) and high risk scores based on Strain Index ( $n=26$ Right UL; $n=7$ Left $U L)$. Instrumentation showed details of recurrency, of postures and of force, which can be used for intervention. Conclusions: It's necessary to develop ergonomic strategies and approaches on WRMSDs prevention (risk assessment and management) that will lead to changes on workstations and working processes.
\end{abstract}

Keywords: Occupational Health, Ergonomics, Work-related Musculoskeletal Disorders (WRMSD), Meatpacking, Occupational Risk Assessment and Management.
Revisado: $25 / 05 / 2009$

Aprovado: 29/05/2009 


\section{Introdução}

Em Portugal, a associação entre trabalho e lesões músculo-esqueléticas (lesões músculo-esqueléticas ligadas $^{5}$ ao trabalho - LMELT ou LMEMSLT - do membro superior $)^{6}$ faz-se quase sempre na indústria automobilística ou nas suas indústrias satélites e mesmo assim provavelmente subavaliadas e subnotificadas. Omitemse (ou esquecem-se) todas as actividades em que existem cadências impostas, com reduzidos ciclos de trabalho - repetitividade -, aplicação de força, posturas extremas a nível dos membros superiores e exposição a vibrações mão-braço que determinam uma probabilidade acrescida de aparecimento (ou de desenvolvimento) das LMEMSLT. Essas actividades, frequentes em meio industrial (BERNARD, 1997; INSTITUTE OF OCCUPATIONAL MEDICINE, 2001), podem encontrar-se em trabalhos tão diversificados quanto a panificação ou a prestação de cuidados de saúde ou, por exemplo, o abate e a desmancha $^{7}$ industrial de animais para consumo humano (BUCKLE; DEVEREUX, 2002; JUUL-KRISTENSEN et al., 2002; MIRKA, 2005).

De facto, o abate e a desmancha industrial de animais para consumo humano comportam a exposição a factores de risco de natureza profissional que podem determinar efeitos adversos para a saúde de quem, nesse contexto, desenvolve a sua actividade profissional. A importância do problema tornou-se evidente nos anos 80 do século XX, o que, provavelmente, influenciou na emissão das primeiras normas de prevenção de LMELT nesse sector industrial, em 1986, nos EUA (OCCUPATIONAL SAFETY AND HEALTH ADMINISTRATION, 1986; 2004).

O conhecimento das situações de trabalho em empresas de abate e desmancha de carne permite constatar que os sistemas de organização do trabalho eram (e são ainda hoje) essencialmente tayloristas ou para-tayloristas, redutores do valor humano no trabalho, determinando que os trabalhadores estejam expostos durante todo o tempo de trabalho - entenda-se durante a realização da actividade diária - a elevadas cadências de trabalho, a repetidas aplicações de força e ao frequente contacto com ferramentas e equipamentos que os sujeitam à exposição a vibrações (BUREAU OF LABOR STATISTICS, 2002; OCCUPATIONAL SAFETY AND HEALTH ADMINISTRATION, 2004; KANDEL, 2006; LIPSCOMB et al., 2007).

Alguns dados estatísticos, ainda dos Estados Unidos da América (EUA), revelam, na indústria de carnes, indicadores de morbilidade crescentes e com- parativamente mais elevados que em outros tipos de actividade; apenas a título de exemplo, só no ano de 1993 foram notificados 19.300 novos casos de LMELT provenientes dessas indústrias (BUREAU OF LABOR STATISTICS, 1993).

Os indicadores de morbilidade apontam, em 1995, para taxas de prevalência de $13 \%$ em trabalhadores da indústria de carnes nos EUA, o que corresponde a uma frequência 38 vezes mais elevada da prevalência do que nos restantes sectores industriais (BUREAU OF LABOR STATISTICS, 1995). Trata-se de indicadores de morbilidade que colocam aquele sector de actividade num elevado grau de prioridade de intervenção correctiva, nem sempre reconhecido em diversos outros países. Mais recentemente, os dados estatísticos de morbilidade por LMELT continuam a evidenciar prevalências elevadas neste sector industrial (BUREAU OF LABOR STATISTICS, 2002; OSHA, 2004; WATERS, 2004; SORMUNEN, 2006).

A actividade de trabalho dos magarefes ${ }^{8}$ exige aplicações de força enquanto se corta a carne (ou se manipulam as peças) em posições articulares extremas a nível de todo o membro superior, em particular do punho/mão, assim como uma elevada repetitividade gestual (MAGNUSSON et al., 1987; MIRKA, 2005). Estima-se, por exemplo, que cada trabalhador execute, em média, cerca de 18 mil movimentos por membro superior no "corte ou na desmancha", num ciclo de trabalho de oito horas (ROMQUIST; HANSSON, 1979; MAGNUSSON et al., 1987; COOK et al., 1999).

Apesar de se conhecerem diversos factores de risco na génese das LMEMSLT, de que se destacam os factores de risco da actividade (frequentemente designados como factores de risco ergonómicos), os factores de natureza individual e os factores de risco psico-organizacionais, a aplicação de medidas de prevenção envolve sempre aspectos de grande complexidade (SERRANHEIRA; UVA, 2002). Essa complexidade reside, desde logo, no frequente desconhecimento (ou pelo menos insuficiente conhecimento) sobre o nível de exposição abaixo do qual não se observam efeitos adversos (NOAEL - no observed adverse effect level) (UVA; GRAÇA, 2004; UVA, 2006) na exposição a factores de risco, mesmo quando considerados isoladamente (WESTGAARD; WINKEL, 1996).

Qualquer intervenção preventiva nas situações de trabalho com risco de LMEMSLT pressupõe uma correcta avaliação do risco, que pode ser realizada através de diversos métodos que vão desde a observação (mais ou menos estruturada) a outros métodos, bem mais complexos, por exemplo, de medida de variáveis

\footnotetext{
${ }^{5}$ Em Portugal, utiliza-se a terminologia "doenças ligadas ao trabalho" que pretende ser abrangente e incluir as doenças relacionadas e agravadas pelo trabalho, assim como as decorrentes, por exemplo, de acidentes de trabalho.

${ }^{6}$ No Brasil, Lesões por Esforços Repetitivos - LER ou Distúrbios Osteomusculares Relacionados com o Trabalho - DORT.

${ }^{7}$ Desmanchar é o processo de preparar, separar e cortar as diferentes partes do animal abatido.

${ }^{8}$ Magarefes são os trabalhadores da indústria de abate e desmancha de carnes em Portugal.
} 
fisiológicas e/ou biomecânicas (SERRANHEIRA; UVA; LOPES, 2008).

O presente estudo, realizado em contexto de trabalho real, teve como principal objectivo testar uma estratégia de diagnóstico do risco e de vigilância da saúde dos trabalhadores numa empresa de abate e desmancha de carne para consumo humano, passando por:

1. estudo da situação real de trabalho (análise da actividade de trabalho);

2. vigilância da saúde dos trabalhadores;

3. identificação dos factores de risco de LMELT;

4. avaliação do risco de LMELT.

\section{Métodos}

O estudo incidiu sobre trabalhadores de uma indústria de abate e desmancha industrial de animais para consumo humano, num total de 56, dos quais 19 do sexo masculino, pretendendo-se:

- identificar sintomatologia de LMELT referida pelos trabalhadores e avaliar clinicamente os trabalhadores sintomáticos;

- diagnosticar as situações de risco dessas lesões, utilizando o filtro RSI risk filter e o método Strain Index (SI) de avaliação de risco de lesões músculo-esqueléticas a nível do membro superior (LMEMSLT);

- identificar aspectos da exposição biomecânica em alguns postos de trabalho, com recurso à instrumentação (electrogoniometria e sensores de força).

Os trabalhadores foram inquiridos, através da versão modificada do Questionário Nórdico Músculo-Esquelético (QNM), aplicado pelos investigadores individualmente a cada trabalhador, sobre (1) as suas características sócio-demográficas; (2) a presença de sintomas em pelo menos quatro dias seguidos nos últimos 12 meses na coluna cervical, ombros, cotovelos, punhos/mãos, coluna dorsal, coluna lombar, ancas/coxas, pernas/joelhos e tornozelos/pés. A intensidade do desconforto (ou dor) foi medida numa escala de 1 a 4 (1 - pequena intensidade; 4 -maior intensidade); e (3) a caracterização da exposição biomecânica na actividade desenvolvida, como: (i) a percentagem de tempo de trabalho em que estavam em pé; (ii) se deambulavam; (iii) se inclinavam ou rodavam o tronco; (iv) se mantinham os membros superiores elevados; (v) se faziam movimentos repetitivos; (vi) se faziam movimentos de precisão ou de força com as mãos ou dedos; (vii) tempo de utilização de facas; (viii) levantamento de cargas; (ix) contacto com ferramentas vibratórias; ( $\mathrm{x}$ ) percentagem de tempo em que estavam em stress e (xi) avaliação da dificuldade em realizar o trabalho.
Os trabalhadores que referiram sintomatologia foram novamente inquiridos sobre a presença de sintomas em pelo menos quatro dos últimos sete dias (avaliação da fiabilidade da resposta). Os trabalhadores que apresentaram sintomatologia no momento e aqueles em que a sintomatologia esteve presente em pelo menos durante quatro dias nos últimos sete dias (critérios de diagnóstico por sintomas), foram, em seguida, avaliados clinicamente por um médico especialista em Reumatologia.

A etapa seguinte do processo de diagnóstico passou pela identificação de factores de risco através do filtro RSI risk filter (GRAVES et al., 2004) e da aplicação seguida de um método de avaliação integrada do risco de LMEMSLT no sentido de classificar e hierarquizar o risco dos diversos postos de trabalho. Utilizou-se o método Strain Index (MOORE; GARG, 1995) devido à sua validação ter sido realizada em contextos de trabalho semelhantes. O método Strain Index engloba a medição (ou a estimativa) de seis variáveis da tarefa: intensidade do esforço; duração do esforço por ciclo de trabalho; número de esforços por minuto; postura da mão/punho; velocidade de trabalho; duração diária da tarefa. Tanto o filtro RSI, como o método SI foram aplicados no local de trabalho por um ergonomista com experiência na sua utilização e, complementarmente, através do visionamento de registros de vídeo de todos os postos de trabalho.

Na última etapa (instrumentação), foram seleccionados, por conveniência devido a limitações várias, postos de trabalho de risco elevado de LMEMSLT com alta repetitividade e aplicação de força. Utilizou-se instrumentação constituída por electrogoniómetros biaxiais SG75 e SG65 da Biometrics ${ }^{\circledR}$ e sensores de força da Flexiforce $^{\circledR}$ de 0 a 440 Newtons e hardware ProComp Infiniti $^{\circledR}$ de 8 canais e software da Ergonomics suite Infiniti Throught Tchnologies ${ }^{\circledR}$. A electrogoniometria do punho/mão direita e a aplicação de sensores de força em 4 dedos foi realizada, em cada posto, pelo menos em duas avaliações de, no mínimo, dois minutos consecutivos em cada setup.

A utilização daqueles equipamentos pretendeu obter uma análise rigorosa dos aspectos cinemáticos e cinéticos de componentes biomecânicas do movimento da mão dominante (BAO; HOWARD; SILVERSTEIN, 2006), designadamente a flexão/extensão e desvios cubitais e radiais a nível da articulação do punho/mão direito, e os níveis de força aplicada por cada um dos primeiros quatro dedos da mão direita, nos postos de trabalho referidos. Para tal, utilizaram-se igualmente registos em vídeo da actividade de trabalho, com recurso a uma câmara vídeo Sony DCE-HC14E ${ }^{\circledR}$, sincronizados com as componentes instrumentais.

Todos os aspectos éticos foram devidamente salvaguardados e todos os trabalhadores envolvidos no estudo foram previamente informados. Os participantes foram voluntários e conscientes da sua participação. Salvaguardaram-se todos os aspectos relativos à Decla- 
ração de Helsínquia. O delineamento do estudo foi submetido a apreciação e aprovado pela Escola Nacional de Saúde Pública - Portugal.

\section{Resultados}

Toda a actividade é desenvolvida numa nave ${ }^{9}$, onde estão montadas as várias linhas de abate e produção organizadas da seguinte forma: abate e esfola de bor$\operatorname{regos}^{10}$, abate e desmancha de porcos, triparia, salsicharia, sala da banha, fiambre e bacon e embalamento. Também existem várias câmaras frigoríficas, onde são armazenados animais inteiros bem como os seus subprodutos.

Os trabalhadores têm um horário de 40h de trabalho semanal com a seguinte distribuição: de $2^{\mathrm{a}}$ a $5^{\mathrm{a}}$ feira das 7:30h às 17:30h, com um intervalo de 15 minutos às 9:15h e de 1 hora para almoço, das 12:30h às 13:30h; $6^{\text {a }}$ feira das 8:00h às 12:00h; descanso semanal ao sábado e domingo.

Os postos de trabalho exigem que o trabalhador permaneça na posição de pé. A maioria dos trabalhadores é polivalente, desempenhando quase todo o tipo de tarefas de cada posto de trabalho, consoante a necessidade de recursos. Mudam com frequência de posto de trabalho, voluntária ou involuntariamente, em função do ciclo de produção ou de necessidades extemporâneas.
O ritmo de trabalho é imposto aos trabalhadores e é marcado pela cadência de abate, aproximadamente 3 animais por minuto em ritmo habitual (tempo de ciclo aproximadamente de 20 segundos), podendo chegar aos 5 animais por minuto (tempo de ciclo de 12 segundos) em épocas de maior consumo, como são os exemplos das épocas do Natal e da Páscoa.

Dos 56 trabalhadores, estudaram-se 50, 33 do sexo feminino (66\%), com uma média de idades de 39 anos (mínimo de 18 e máximo de 61 anos), altura média de $164 \mathrm{~cm}$ (mínimo de $150 \mathrm{~cm}$ e máximo de $187 \mathrm{~cm}$ ) e peso médio de $69 \mathrm{~kg}$ (mínimo de $49 \mathrm{~kg}$ e máximo de $100 \mathrm{~kg}$ ). A antiguidade dos trabalhadores na empresa tem uma média de 10 anos, variando entre os 2 meses e os 25 anos.

Nos últimos 12 meses (Tabela 1), 41 trabalhadores (82\%) referiram sintomas músculo-esqueléticos ligados ao trabalho por, pelo menos, 4 dias seguidos, enquanto os restantes $9(18 \%)$ negam qualquer sintomatologia. As queixas da coluna lombar foram referidas por 32 (64\%) e dos punhos/mãos por 27 (54\%). Quanto à intensidade média das queixas, verificou-se que é mais elevada nos cotovelos $(2,8)$, seguida dos punhos/mãos e pernas/joelhos $(2,5)$. Coluna cervical, ombros, coluna lombar e tornozelos/pés apresentaram a mesma intensidade média: 2,2 .

A presença de sintomatologia no momento de resposta, considerando pelo menos 4 dias de sintomas durante os últimos 7 dias (sintomas recentes na Tabela 1), foi referida por 30 inquiridos (60\%), estando ausen-

Tabela 1 Trabalhadores de indústria de abate e desmancha de carne, com sintomatologia e sua intensidade

\begin{tabular}{lccc}
\hline & \multicolumn{2}{c}{$\begin{array}{c}\text { Sintomas } \\
\text { últimos } 12 \text { meses }\end{array}$} & $\begin{array}{c}\text { No de trabalhadores com } \\
\text { sintomas recentes }\end{array}$ \\
& $N^{0}$ de & Intensidade \\
trabalhadores & média & \\
\hline Coluna cervical & 4 & 2,2 & 2 \\
Ombros & 6 & 2,2 & 3 \\
Cotovelos & 6 & 2,8 & 6 \\
Punhos/Mãos & 27 & 2,5 & 19 \\
Coluna Dorsal & 0 & - & 0 \\
Coluna Lombar & 32 & 2,2 & 20 \\
Ancas/Coxas & 0 & - & 2 \\
Pernas/Joelhos & 4 & 2,5 & 2 \\
Tornozelos/Pés & 4 & 2,2 & 54 \\
\hline Total & 83 & - & \\
\hline
\end{tabular}

\footnotetext{
${ }^{9}$ Galpão industrial.

${ }^{10} \mathrm{~A}$ esfola de borregos é um dos postos de trabalho onde se retira a pele do animal. Borrego: carneiro pequeno.
} 
te em $20(40 \%)$. As regiões referidas como tendo sintomas foram, no essencial, a coluna lombar (20) e os punhos/mãos (19).

Foram observados clinicamente os 20 trabalhadores com sintomas nos cotovelos e punhos/mãos (15 do sexo feminino e 5 do sexo masculino), sendo possível diagnosticar patologia músculo-esquelética em 15. São 30\% do total dos trabalhadores incluídos no estudo (13 em 33 do sexo feminino - 40\% e 2 em 17 do sexo masculino $-12 \%)$. Os 5 restantes apresentavam queixas de parestesias nas mãos.

Com base em critérios clínicos, foram diagnosticadas, pelo médico reumatologista, patologias, de que se destacam a Síndrome do Canal Cárpico (SCC) e a artrose dos dedos (Tabela 2).

Destaca-se igualmente que a presença de sintomatologia distal dos membros superiores nos últimos 12 meses e nos últimos 7 dias está ligada à actividade desenvolvida (trabalho efectuado), existindo: (i) uma associação entre a presença de sintomas nos cotovelos, no último ano e no presente, com a esfola de borregos ( $p<0,05)$; (ii) uma associação entre a presença de sintomas nos punhos/mãos nos últimos 12 meses e a esfola de borregos, levantamento/empurrar cargas $<18$ $\mathrm{kg}$ e utilização de ferramentas vibratórias $(p<0,05)$; (iii) uma associação entre a presença de sintomatologia actual dos punhos/mãos e a esfola de borregos e a utilização de ferramentas vibratórias $(p<0,05)$; e (iv) uma associação entre a presença de lesão e a esfola de borregos, actividades que incluem movimentos de precisão com os dedos e utilização de ferramentas vibratórias $(p<0,05)$.

A aplicação do filtro RSI (GRAVES et al., 2004) permitiu identificar a presença de sinais ou sintomas de LMELT em 23 dos 49 postos de trabalho. Identificou-se a presença de repetitividade em 31 postos de trabalho, sete dos quais com repetitividade muito elevada. Identificaram-se apenas algumas situações de trabalho em que a postura não foi considerada como de risco $(n=9)$. Nos restantes postos de trabalho, observou-se principalmente a presença de posturas extremas ou fora dos ângulos intersegmentares de conforto $(n=26)$. A aplicação de força identificou-se em todos os postos de trabalho $(\mathrm{n}=49)$. Destaca-se a aplicação de força durante a acção de segurar a faca no processo de corte e desmancha de carne $(n=20)$. Seguem-se as acções de agarrar e de aplicar força repetidamente $(n=8)$. Identificou-se, por fim, o contacto com equipamentos ou ferramentas vibratórias em 3 postos de trabalho.

Os resultados da aplicação do método Strain Index (SI) (MOORE; GARG, 1995) em cada posto de trabalho identificaram níveis de risco consideráveis (e necessidades de intervenção) num elevado número de postos com alto risco de LMEMSLT. Cada aplicação do método foi efectuada bilateralmente de modo a permitir identificar o nível de risco em cada membro superior (Gráfico 1). Destacam-se 26 postos de trabalho com níveis de risco elevados e, entre esses, 5 postos de trabalho têm scores SI superiores a 20 e 2 deles superiores a 50 .

A análise dos resultados permite constatar que os elementos da situação de trabalho mais implicados na determinação dos scores são as aplicações de força muito elevadas $(n=12)$, com nível 6 na escala CR10 de Borg (BORG, 1998), a realização de esforço durante pelo menos $80 \%$ do tempo do ciclo $(n=8)$ e a repetitividade gestual com mais de 20 aplicações de força por minuto $(\mathrm{n}=28)$.

Dos postos identificados como de risco muito elevado, seleccionaram-se, por conveniência, quatro postos de trabalho de elevada repetitividade e aplicação de força, onde se utilizaram componentes instrumentais, designadamente a electrogoniometria no punho/mão direita e a aplicação de sensores de força (nos 4 primeiros dedos da mão direita).

Identificaram-se distintos padrões gestuais e de aplicação de força dos postos analisados. No essencial,

Tabela 2 Patologias diagnosticadas por exame clínico, em trabalhadores de indústria de abate e desmancha de carne

\begin{tabular}{lc}
\hline \multicolumn{1}{c}{ Patologias diagnosticadas } & Casos \\
\hline Síndrome do Canal Cárpico (SCC) & 9 \\
Artroses dos dedos & 5 \\
Epicondilites & 4 \\
Doença de De Quervain & 2 \\
Síndrome do Canal de Guyon & 1 \\
Tendinite dos Radiais & 1 \\
Tenoperiostite do Grande Palmar & 1 \\
Sinovite dos dedos & 1 \\
\hline Total & 24 \\
\hline
\end{tabular}


os registos permitiram verificar predomínios de repetitividade gestual que oscilam entre pequenas e grandes amplitudes intersegmentares e a prevalência de extensão e desvio cubital do punho.

Observou-se, por exemplo, no posto de "preparação das lombadas ${ }^{11}$ ", um padrão de realização da actividade de trabalho com picos de extensão do punho/mão (Figuras 1 e 2) até aproximadamente $30^{\circ}\left(\min .27,22^{\circ}\right.$ e máx. $31,24^{\circ}$, repetindo-se ciclicamente em cada 20 segundos) durante o início do corte longitudinal dos tecidos que permitem separar o membro anterior do animal da respectiva carcaça.

A repetitividade situou-se acima dos 70 movimentos (cerca de 35 acções técnicas) por minuto a nível daquela articulação e, por consequência, encontra- -se epidemiologicamente num nível de risco elevado de LMEMSLT.

Relativamente às posições de desvios do punho (radiais e cubitais), é notória a predominância de desvio cubital, apesar de, em média, se situar apenas nos 7,4 ${ }^{\circ}$ (considerado aceitável ou de baixo risco). Observam-se, apesar disso, um conjunto de movimentos repetitivos entre os $0^{\circ}$ a $5^{\circ}$ e os $15^{\circ}$ a $20^{\circ}$ de desvio cubital, resultados já considerados de risco moderado de LMEMSLT.

Relativamente à aplicação de força a nível dos dedos/mão direitos, observa-se, neste exemplo, um predomínio de aplicação de força com o $1^{\circ}$ dedo com registos que, em média, se aproximam de valores de força da ordem dos 6,5 N. Apesar disso, existem frequentes picos de força de aproximadamente $30 \mathrm{~N}$ e, a nível do $1^{\circ}$ e $4^{\circ}$ dedos, repetidos com uma frequência de mais de 2

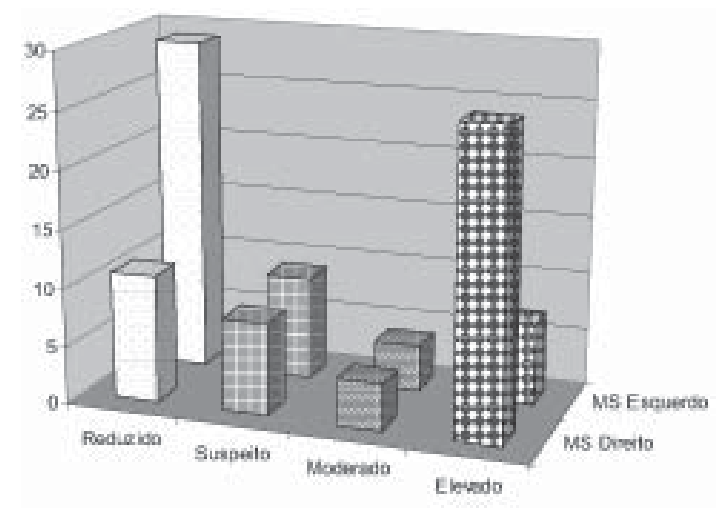

Gráfico 1 Scores SI - Classificação do risco de LMELT nos postos de trabalho de abate e desmancha de carne

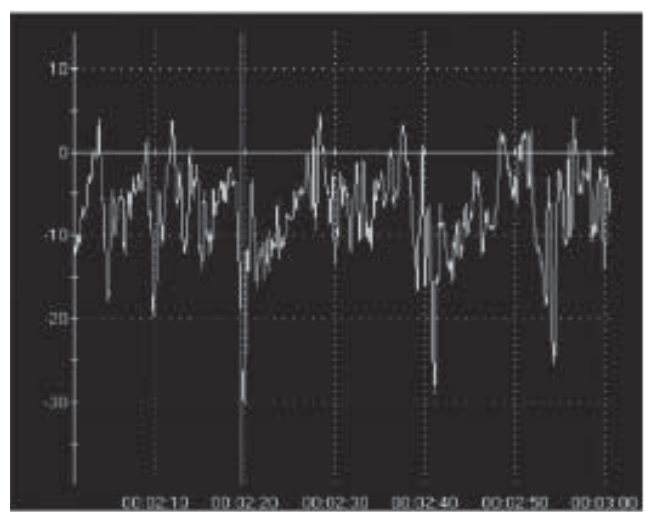

Figura 1 Registo gráfico representativo dos movimentos de extensão/flexão da mão/punho direito, na actividade de preparação das lombadas

\footnotetext{
${ }^{11}$ Corte realizado para separar o tecido da respectiva carcaça.
} 


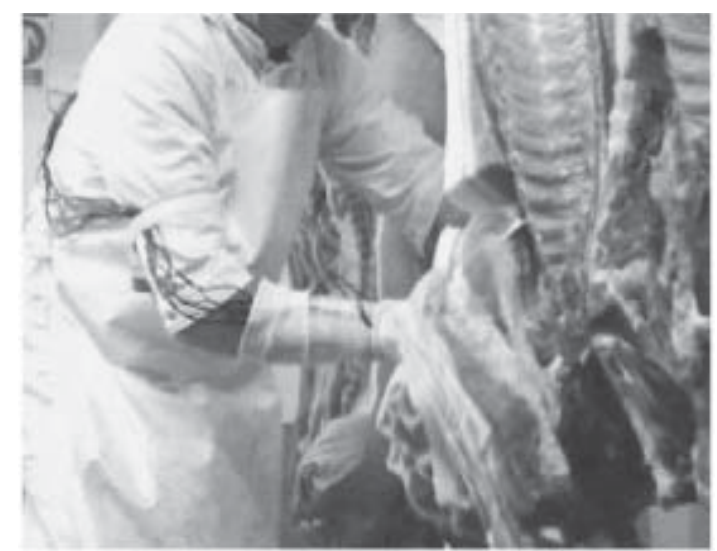

Figura 2 Exemplo de instrumentação no posto de preparação das lombadas

vezes em cada 10 segundos. Esses registos de aplicação de força podem ser considerados como indutores de uma elevada carga física de trabalho localizada e, por consequência, da eventual presença de níveis de fadiga muscular localizada.

\section{Discussão e conclusões}

Trata-se de uma população que é maioritariamente do sexo feminino (66\%) com uma média de idade de 39 anos que, subjectivamente, caracteriza a dificuldade do seu trabalho no dispêndio de esforço, ainda que os postos de trabalho envolvam essencialmente a repetitividade. As trabalhadoras do sexo feminino referem, nos últimos 12 meses, queixas nos punhos/mãos (52\%), ainda que a sintomatologia nos cotovelos (18\%) e as queixas na coluna cervical (12\%) também sejam mencionadas. Estes resultados são menos expressivos do que os obtidos em anteriores estudos por nós realizados em indústrias com actividades semelhantes (SERRANHEIRA et al., 2003), em que são referidas prevalências superiores: punhos/mãos (84\%), coluna cervical $(71 \%)$, ombros $(62 \%)$ e cotovelos $(70 \%)$. Outros estudos mais recentes evidenciam igualmente prevalências de sintomas músculo-esqueléticos preocupantes nesta indústria (KANDEL, 2006; LIPSCOMB, 2007).

Existirão, por certo, inúmeras razões evocáveis para a diferença de resultados assinalada, destacando-se, para além da diferença nos postos de trabalho e trabalhadores, a forma de aplicação dos questionários. Apesar disso, destaca-se que, entre os trabalhadores sintomáticos a nível do punho/mão, quer nos últimos 12 meses, quer nos últimos 7 dias, existe um significativo número de casos diagnosticados $(p<0,01)$. Tal facto pode dever-se a uma aplicação do questionário apoiada pelos investigadores, o que se considera ser um contributo para uma maior objectividade das respostas.

Em 15 dos 20 trabalhadores (75\%) com sintomatologia actual, diagnosticou-se, clinicamente, pelo menos uma lesão músculo-esquelética, o que perfaz 30\% do número total de trabalhadores, valores semelhantes aos obtidos por Viikari-Juntura (1983). A presença de lesão é muito mais prevalente no sexo feminino (39\%) do que no masculino - $(12 \%)$.

Mais de $80 \%$ dos trabalhadores referiram sintomatologia por pelo menos 4 dias seguidos nos últimos 12 meses. Ou, se se preferir, apenas nove trabalhadores negam sintomatologia naquele período de tempo. A maior intensidade das queixas verifica-se nos cotovelos e punhos e nas mãos. Sessenta por cento refere sintomatologia por pelo menos 4 dias nos últimos 7 , mais localizada à coluna lombar e punhos e mãos. Trata-se de valores muito expressivos e bem representativos do grau de prioridade que deve ser atribuída à implementação de medidas preventivas.

A lesão mais prevalente foi a síndrome do canal cárpico (SCC), mais frequente no sexo feminino (SARAIVA, 1995; LEWCZUK; AFFELSKA-JERCHA, 2002), que foi diagnosticada em 9 trabalhadores, sendo 8 do sexo feminino. Esses índices de morbilidade (16\%) eram esperados, uma vez que outros estudos referem valores compreendidos entre $10 \%$ e $25 \%$ (FALK; AARNIO, 1983; PUJOL, 1993; LEWCZUC, 2002). As artroses dos dedos $(n=5)$ e epicondilites $(n=4)$ também foram lesões muito frequentes.

A metodologia diagnóstica de avaliação do risco de LMEMSLT (identificação de factores de risco e avaliação do risco) revelou a exposição frequente aos principais factores de risco (postura extrema, repetitividade e aplicação de força) e níveis de risco elevados predominantemente no membro activo, destacando-se, nesse contexto, cinco valores de SI superiores a vinte, dos quais dois superiores a 50. Trata-se de resultados que determinam urgência na introdução de medidas de intervenção susceptíveis de gerir tão significativos níveis e risco.

A electrogoniometria e a aplicação de sensores de força, com registos vídeo sincronizados, permitiram a obtenção de informação complementar no que concerne ao método observacional de avaliação do ris- 
co utilizado, designadamente a nível dos momentos efectivos de aplicação de força, repetitividade, posturas e amplitude dos movimentos. A sua realização, em quatro postos de trabalho com risco elevado de LMEMSLT, revela, apenas a nível exploratório, distintos padrões gestuais e de aplicação de força, com predomínio para as amplitudes intersegmentares e a repetitividade. Tais resultados poderão contribuir para uma mais adequada emissão de propostas de intervenção correctiva sobre o trabalho (entenda-se, por exemplo, intervenção a nível dos postos de trabalho e dos meios técnicos utilizados e a nível da organização do trabalho), fundamentais para a prevenção das LMELT.

\section{Referências}

BAO, S.; HOWARD, P.; SILVERSTEIN, B. Quantifying repetitive hand activity for epidemiological research on musculoskeletal disorders - part II: comparison of different methods of measuring force level and repetitiveness. Ergonomics, England, v. 49, n. 4, p. 381392, 2006.

BERNARD, B. (Ed.). Musculoskeletal disorders and workplace factors: a critical review of epidemiologic evidence for work-related musculoskeletal disorders of the neck, upper extremity and low back. Cincinnati: NIOSH, 1997.

BORG, G. Borg's perceived exertion and pain scales. Champaign: Human Kinetics, 1998.

BUCKLE, P. W.; DEVEREUX, J. J. The nature of work-related neck and upper limb musculoskeletal disorders. Applied Ergonomics, England, v. 33, n. 3, p. 207-217, 2002.

BUREAU OF LABOR STATISTICS. Occupational injuries and illnesses in the United States by industry. Washington: BLS, 1993.

BUREAU OF LABOR STATISTICS. Occupational injuries and illnesses - industry data (1989-2001): poultry slaughtering and processing. Washington: BLS, 2002.

COOK, T. et al. Electromyographic effects of ergonomic modifications in selected meatpacking tasks. Applied Ergonomics, England, v. 30, n. 3, p. 229233, June,1999.

FALK, B.; AARNIO, P. Left-sided carpal tunnel syndrome in butchers. Scandinavian Journal of Work Environment \& Health, Finland, v. 9, n. 3, p. 291-297, 1983.

GRAVES, R. J. et al. Development of risk filter and risk assessment worksheets for HSE guidance - Upper limb disorders in the workplace 2002. Applied Ergonomics, England, v. 35, n. 5, p. 475-484, 2004.
No presente estudo optou-se por testar um conjunto de etapas sequenciais, desde a análise das situações de trabalho, identificação de sintomas, avaliação clínica de sinais, diagnóstico do risco (identificação de factores de risco e avaliação do risco) que, quando aplicados de forma integrada e interpretados com rigor e por técnicos com formação no domínio da Ergonomia, Higiene e Segurança do Trabalho, possibilitam conhecer melhor o "ambiente" de trabalho e, dessa forma, permitem valorizar as necessidades de intervenção sobre os sistemas de trabalho, melhorando-os e tornando-os mais adaptados ao trabalhador e, por consequência, mais produtivos.
INSTITUTE OF OCCUPATIONAL MEDICINE.

Musculoskeletal disorders and the workplace: low back and upper extremities. Washington, National Academy Press, 2001.

JUUL-KRISTENSEN, B. et al. Physical workload during manual and mechanical deboning of poultry. International Journal of Industrial Ergonomics, English, v. 29, n. 2, p. 107-115, 2002.

KANDEL, W. Meat processing firms attract Hispanic workers to rural America. In: Amber Waves: the economics of food, farming, natural resources and rural America. 2006. Disponível em: < http:// www.ers.usda.gov/AmberWaves/june06/features/ meatProcessing.htm>. Acesso em: 8 nov. 2008.

LEWCZUK, E.; AFFELSKA-JERCHA, A. Occupational and non-occupational aspects of carpal tunnel syndrome. Medycyne Pracy, Poland, v. 53, n. 5, p. 417422, 2002.

LIPSCOMB, H. et al. Musculoskeletal symptoms among poultry processing workers and a community comparison group: black women in low-wage jobs in the rural south. American Journal of Industrial Medicine, United States, v. 50, n. 5, p. 327-338, May 2007.

MAGNUSSON, M. et al. An ergonomic study of work methods and physical disorders among professional butchers. Applied Ergonomics, England, v. 18, n. 1, p. 43-50, 1987

MIRKA, G. A. Development of an ergonomics guideline for the furniture manufacturing industry. Applied Ergonomics, England, v. 36, n. 2, p. 241-247, 2005.

MOORE, J.; GARG, A. The strain index: a proposed method to analyse jobs for risk of distal upper extremity disorders. American Industrial Hygiene Association Journal, United, States, v. 56, n. 5, p. 443458, May 1995. 


\section{NATIONAL INSTITUTE OF OCCUPATIONAL}

SAFETY AND HEALTH. Cumulative trauma disorders in the workplace: bibliography. Cincinnati: NIOSH, 1995.

\section{OCCUPATIONAL SAFETY AND HEALTH}

ADMINISTRATION. Ergonomics program management guidelines for the meatpacking industry. Arlington Heights: OSHA, 1986.

\section{OCCUPATIONAL SAFETY AND HEALTH}

ADMINISTRATION. Guidelines for poultry processing: ergonomics for the prevention of musculoskeletal disorders. Arlington Heights: OSHA, 2004.

PUJOL, M. Pathologie professionnelle d'hypersolicitation - atteinte périarticulaire du membre supérieur. Paris : Masson, 1993. (Collection de Monographies de Médicine du Travail)

ROMQUIST, S.; HANSSON, J. Occupational disorders among butchers: Investigation of butcher's working conditions. Stockholm: Arbetarskydsstyrelsen Report, 1979.

SARAIVA, F. Síndromas canaliculares. Cadernos de Reumatologia, n. 6, p. 73-99, 1995.

SERRANHEIRA, F. ; UVA, A. Lesões músculoesqueléticas ligadas ao trabalho (LMELT): aspectos gerais de diagnóstico e prevenção. In: UVA A. NETO, L. MIRANDA, L. [Ed.]. Doenças reumáticas ligadas ao trabalho. Lisboa: Liga Portuguesa Contra as Doenças Reumáticas, Instituto de Desenvolvimento e Inspecção das Condições de Trabalho, 2002.

SERRANHEIRA, F. et al. Auto-referência de sintomas de Lesões músculo-esqueléticas e trabalho (LMELT) numa grande empresa em Portugal. Revista Portuguesa de Saúde Pública, Lisboa, v. 21, n. 2, p. 37-48, jul./dez. 2003.

SERRANHEIRA, F.; UVA, A.; LOPES, F. Lesões músculo-esqueléticas e trabalho: alguns métodos de avaliação do risco. Lisboa: Sociedade Portuguesa de Medicina do Trabalho, 2008. (Cadernos Avulso, 5)

SORMUNEN, E. et al. Muscular and cold strain of female workers in meatpacking work. International Journal of Industrial Ergonomics, Netherlands, v. 36, n. 8, p. 713-720, 2006.

UVA, A. Diagnóstico e gestão do risco em saúde ocupacional. Lisboa: ISHST, 2006. (Segurança e Saúde no Trabalho. Estudos, 17)

UVA, A.; GRAÇA, L. Glossário de saúde e segurança do trabalho. Lisboa: Sociedade Portuguesa de Medicina do Trabalho, 2004 (Cadernos Avulso, 4)

VIIKARI-JUNTURA, E. Neck and upper limb disorders among slaughterhouse workers. Scandinavian Journal of Work, Environment \& Health, Finland, n. 9, p. 283290,1983

WATERS, T. R. National efforts to identify research issues related to prevention of workrelated musculoskeletal disorders. Journal of Electromyography and Kinesiology, England, v. 14, n. 1, p. 7-12, Feb. 2004.

WESTGAARD, R. H.; WINKEL, J. Guidelines for occupational musculoskeletal load as a basis for intervention: a critical review. Applied Ergonomics, England, v. 27, n. 2, p. 79-88, Apr. 1996. 